\title{
Thermal Plasma Flow and Equivalent Circuit Analyses on the Electrical Coupling of a $D C-R F$ Hybrid Plasma Torch
}

\author{
Jun-Ho Seo, ${ }^{*}$ Jin-Myung PARK ${ }^{\dagger}$ and Sang Hee HonG ${ }^{\ddagger}$ \\ Department of Nuclear Engineering, Seoul National University, Seoul 151-744
}

(Received 6 October 2008)

\begin{abstract}
Numerical analyses on the electrical coupling of a $D C-R F$ (direct current - radio frequency) hybrid plasma torch are conducted on the basis of magneto-hydrodynamic flow and equivalent circuit models to find the dependency of the coupling efficiency on the $R F$ frequency and the confinement tube's radius. Computations are also carried out for the inductively coupled $R F$ plasma torch to make a comparison between the calculated results. Numerical results reveal that the electrical coupling efficiencies of the $R F$ and the $D C-R F$ hybrid plasma torches have a similar dependency on the $R F$ frequency with an almost constant difference of slightly higher efficiencies for the hybrid plasma due to the radially expanded $D C-R F$ hybrid plasma toward the confinement tube's wall compared with the $R F$ plasma. However, the reduction for a confinement tube's radius less than some critical value, for instance $22 \mathrm{~mm}$ in this numerical work, is found to possibly cause the coupling efficiency of the hybrid plasma to drastically deteriorate compared with that of the $R F$ plasma. Such poor efficiency of a hybrid torch with a relatively small radius is attributed to a significant diminution of the high-temperature region upstream between the $D C$ torch's exit and the first induction coil segment. As a result of this reduced high-temperature region, the magnetic flux linkage is decreased for a smaller confinement tube, which leads to a drastic decrease in the electrical coupling. The present numerical analyses indicate that a special focus needs to be brought the influences of the $D C$ arc jet on the electrical and the flow characteristics of a $D C$ - $R F$ hybrid plasma in determining the torch dimensions for effective conversion of the $R F$ power into the plasma.
\end{abstract}

PACS numbers: $52.30 . \mathrm{Cv}, 52.50 . \mathrm{Qt}, 52.75 . \mathrm{Hn}, 52.77 . \mathrm{Fv}$

Keywords: $D C-R F$ hybrid plasma, Coupling efficiency, $R F$ frequency, Confinement tube, MHD flow, Equivalent circuit, Numerical analysis

\section{INTRODUCTION}

By injecting a $D C$ (direct current) arc jet into the centerline of an inductively coupled $R F$ (radio frequency) plasma torch, a $D C-R F$ hybrid plasma torch can produce a large-volume of high-temperature flame, as well as a central column of high-velocity streams without re-circulation eddies [1]. Owing to these unique features in flow fields, the $D C-R F$ hybrid plasma torch has drawn attention to its possible practical applications to plasma synthesis, thermal plasma chemical-vapor deposition (TPCVD), spray coatings and so on [1-5]. If a $D C-R F$ hybrid plasma torch is to be used in a practical manner, however, it should be designed to assure high

\footnotetext{
*Present Address: Cheorwon Plasma Research Institute, Kangwon -do 269-802;

†Present Address: Experimental Plasma Physics, Fusion Energy Division, Oak Ridge National Laboratory, Oak Ridge, TN 37831, U.S.A.;

‡E-mail: hongsh@snu.ac.kr; Fax: +82-2-889-2688;
}

electrical coupling efficiency, which is defined as the ratio of Joule heat dissipated in the plasma to the total magnetic energy available in the torch, due to its inductively coupled $R F$ torch. As a standard plasma source extensively used in numerous thermal plasma applications, the $R F$ plasma torch can be designed to maximize the values of this coupling efficiency by optimizing the torch dimensions based on the simple scaling law between the electrical skin depth and the plasma radius [6, 7]. Moreover, this scaling law can be obtained normally by assuming the $R F$ plasma to be a cylindrical conductor and by applying the conventional induction heating theory to the simplified plasma [6-10]. In the $D C-R F$ hybrid plasma, however, it is inappropriate to assume the plasma to be a simple cylindrical conductor because the shape of the hybrid plasma in the induction coil region is significantly affected by the superposition of the $D C$ arc jet on the $R F$ plasma [1]. This implies that there is no simple scaling law for an electrically optimized design of the $D C-R F$ hybrid torch; consequently, the electrical coupling efficiency of the $D C-R F$ hybrid plasma should 
be examined by taking into account its complicated flow fields simultaneously. Recently, owing to developments in numerical impedance calculation methods [11-14], numerical techniques have been advanced in dealing with $R F$ plasmas to calculate not only their flow fields but also equivalent electrical parameters. Since the electrical coupling efficiency is expressed in terms of the equivalent circuit parameters from basic electrical circuit theory, these numerical techniques can be used for calculating the efficiency of a $D C-R F$ hybrid plasma, together with its flow fields.

The aim of the present paper is to apply this numerical impedance calculation method to the numerical model for a $D C$ - $R F$ hybrid plasma and to clarify the dependency of the electrical coupling efficiency on the torch design parameters, especially the $R F$ frequency and the confinement tube's radius, for an electrically optimized torch design. For this purpose, firstly, an integrated numerical model of the $D C$ - RF hybrid plasma is presented in this work to compute the $D C$ arc jet and the $R F$ thermal plasma flows simultaneously for the entire region of the $D C-R F$ hybrid plasma torch, including the inside of the $D C$ torch. This integrated mathematical model combining the $D C$ arc jet and the inductively coupled $R F$ plasmas can give more realistic results for flow fields of the hybrid plasma, compared with the conventional approach, which treats the prescribed $D C$ arc jet flows as boundary conditions at the $R F$ torch inlet and solve the related magneto-hydrodynamic (MHD) equations only in a confined region of the $R F$ torch. In the next step, the electrical parameters, such as the equivalent resistance, inductance and electrical coupling efficiency, are calculated for various values of the $R F$ frequency and the confinement tube's radius by combining one of the impedance calculation methods with the proposed integrated model for the $D C-R F$ hybrid plasma. Computations are also conducted for an inductively coupled $R F$ plasma and the calculated results are compared with those for the $D C-R F$ hybrid plasma. Based on the result of this comparative study, the dependency of the electrical coupling efficiency on the $R F$ frequency and the confinement tube's radius is discussed.

\section{MATHEMATICAL FORMULATION OF A THERMAL PLASMA FLOW MODEL}

\section{Torch Geometry, Numerical Method and Ba- sic Assumptions}

Figure 1 shows the computational domains in dotted lines for the $R F$ (left) and the $D C-R F$ hybrid (right) torches. It is seen in the right-hand figure for the $D C$ $R F$ hybrid plasma modeling that the interior region between the conical cathode and the nozzle anode of a $D C$ torch is directly connected with the interior region of a confinement tube for an $R F$ torch. For the comparison

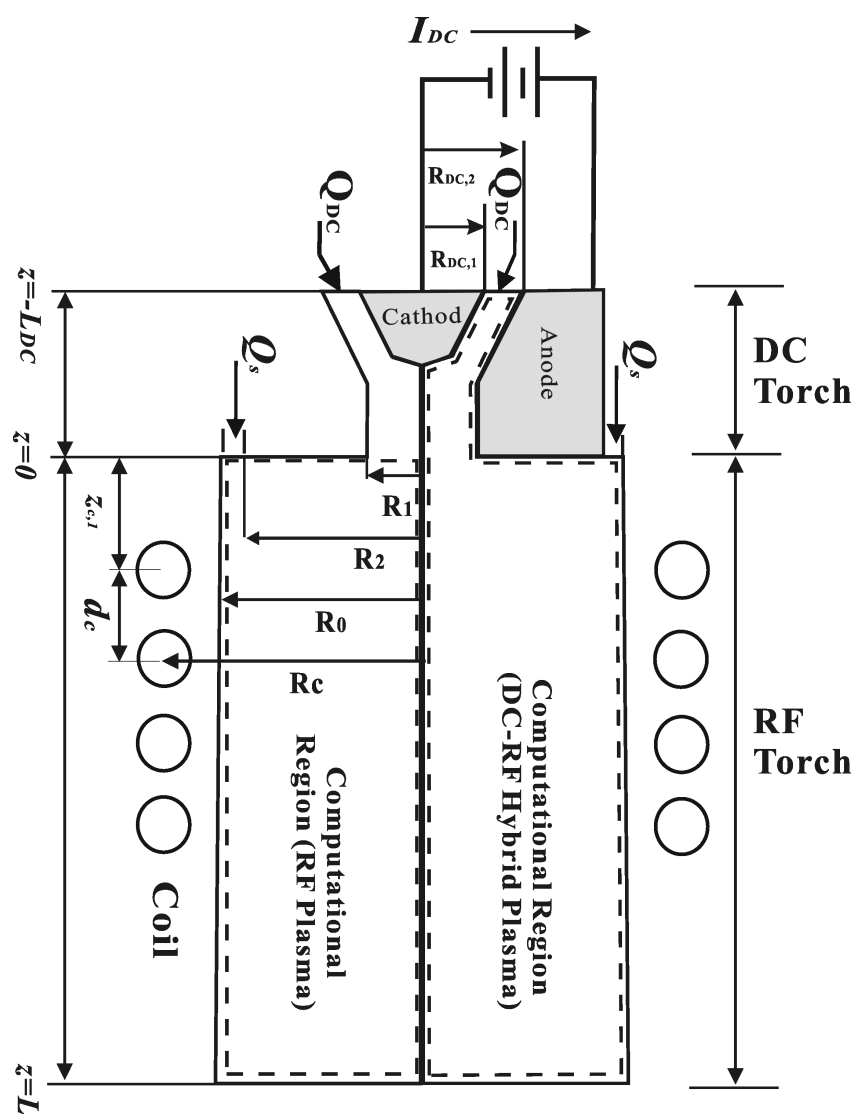

Fig. 1. Schematic diagram of a $D C-R F$ hybrid plasma torch indicating its operation and design parameters, together with computational domains for numerical modeling.

between the $R F$ and the $D C-R F$ hybrid plasmas, the dimensions of the $R F$ part of the hybrid torch have the same values as those of the $R F$ torch depicted in the left part of Figure 1. Plasma forming gases of flow rate $Q_{D C}$ pass through the cathode-anode channel and are ejected from the $D C$ torch nozzle exit, with radius of $\mathrm{R} 1$, into the $R F$ torch and the sheath gas with flow rate Qs for the $R F$ torch is injected around the confinement tube wall from an annular channel between $R_{2}$ and $R_{0}$. The torch dimensions and the operating conditions indicated in Figure 1 are summarized in Table 1 for the present numerical work. The computational domains are divided by rectangular grids and the numbers of mesh cells generated by these grids are $160(20 \times 8)$ and $864(32 \times$ 27 ) inside the $D C$ and the $R F$ torches, respectively. All governing equations are discretized by using the finite volume method (FVM) and are solved using a SIMPLE algorithm [15].

Since the MHD model for the $D C$ arc jet and the $R F$ plasma flows can be described by the continuity, momentum and energy equations, the flow fields of the $D C-R F$ hybrid plasma can be found by solving these conservation equations simultaneously in the entire region shown in Figure 1. In addition, two more mathematical formu- 
Table 1. Input values of the operating and the design parameters indicated in Figure 1 for numerical simulations of the $R F$ and the $D C-R F$ hybrid plasmas.

\begin{tabular}{|c|c|c|}
\hline \multicolumn{2}{|c|}{ Parameters } & Input Values \\
\hline \multirow{4}{*}{ Operation } & $P_{R F}[\mathrm{~kW}]$ & 10 \\
\hline & $Q_{\mathrm{DC}}[\mathrm{lpm}]$ & 4 \\
\hline & $Q_{s}[\mathrm{lpm}]$ & 21 \\
\hline & $I_{D C}[\mathrm{~A}]$ & 0 \\
\hline \multirow{12}{*}{ Design } & $R_{D C, 1}[\mathrm{~mm}]$ & 5 \\
\hline & $R_{D C, 2}[\mathrm{~mm}]$ & 7 \\
\hline & $R_{1}[\mathrm{~mm}]$ & 4 \\
\hline & $R_{0}[\mathrm{~mm}]$ & $17.5,20,22.5,25,30,35$ \\
\hline & $R_{2}[\mathrm{~mm}]$ & $R_{0}-2$ \\
\hline & $R_{c}[\mathrm{~mm}]$ & $R_{0}+10$ \\
\hline & $f[\mathrm{MHz}]$ & $0.5,1,2,3,4,5,6$ \\
\hline & $N$ & 4 \\
\hline & $d_{c}[\mathrm{~mm}]$ & 20 \\
\hline & $Z_{c, 1}[\mathrm{~mm}]$ & 30 \\
\hline & $L_{D C}[\mathrm{~mm}]$ & 35 \\
\hline & $L[\mathrm{~mm}]$ & 150 \\
\hline
\end{tabular}

lations are needed to determine the electromagnetic fields in the $D C-R F$ hybrid plasma. One is the electrostatic potential equation for the $D C$ arc jet and the other is the magnetic vector potential equations, which were developed by Mostaghimi and Boulos [16], for computing the $R F$ electromagnetic fields induced in the hybrid plasma. Since most of $R F$ electromagnetic fields are dissipated in the skin depth of the hybrid plasma located close to the confinement tube's wall and its thickness can be estimated as $<0.01 \mathrm{~mm}$ at the $R F$ frequency of several $\mathrm{MHz}$, the electromagnetic fields induced by the coil currents of the $R F$ torch can be neglected in the $D C$ arc jet generated along the centerline both inside and outside the $D C$ torch. In the proposed model, the magnetic induction and the Joule heat loss in the anode conductors of the $D C$ torch are also negligible because normally $R F$ fields quickly decay if the induction coil is located sufficiently far away from the $D C$ torch exit [17]. Based on these assumptions for electromagnetic interactions in a $D C-R F$ hybrid plasma torch, the electrostatic potential $\phi$ and the magnetic vector potential A can be found separately by solving the respective governing equations over the entire region of the $D C-R F$ hybrid plasma torch. Furthermore, the present numerical model takes into account the following physical assumptions for the hybrid plasma:

- Two-dimensional (2-D) axisymmetric flow and electromagnetic fields,

- A negligible displacement current in the plasma,

- Laminar flow,

- An optically thin plasma,
- Local thermodynamic equilibrium (LTE),

- An argon plasma at atmospheric pressure.

\section{Governing MHD Equations and Boundary Conditions}

\section{A. Electromagnetic Field Equations}

According to the above-mentioned assumptions for the electrical interactions in the $D C-R F$ hybrid plasma torch, the electromagnetic fields and their associated source terms appearing in the MHD flow equations are determined in the computational region of the hybrid torch as follows. Firstly, the electrostatic potential $\phi$ is found from Poisson's equation derived from Gauss's law:

$$
\frac{\partial}{\partial z}\left(\sigma \frac{\partial \phi}{\partial z}\right)+\frac{1}{r} \frac{\partial}{\partial r}\left(\sigma r \frac{\partial \phi}{\partial r}\right)=0
$$

where $\sigma$ is the electrical conductivity. Then, the arc current density, $\boldsymbol{j}$, is calculated inside the $D C$ torch by using

$$
j_{z}=-\sigma \frac{\partial \phi}{\partial z}, \quad j_{r}=-\sigma \frac{\partial \phi}{\partial r} .
$$

The self-induced magnetic field $\boldsymbol{B}^{\boldsymbol{D C}}$ caused by the $D C$ arc current is obtained from Ampere's law, $\nabla \times$ $\boldsymbol{B}^{\boldsymbol{D} \boldsymbol{C}}=\mu_{0} \boldsymbol{j}$. As a result of the generation of these two fields, $\boldsymbol{B}^{\boldsymbol{D C}}$ and $\boldsymbol{j}$, the Lorentz force and the Joule heating generated in the $D C$ arc jet can be calculated by using

$$
\begin{aligned}
& F_{z}^{D C}=-\frac{1}{2} j_{r} B_{\theta}^{D C}, \\
& F_{r}^{D C}=+\frac{1}{2} j_{z} B_{\theta}^{D C}, \\
& P_{j}^{D C}=\frac{1}{2 \sigma}\left[j_{r}^{2}+j_{z}^{2}\right] .
\end{aligned}
$$

In the $R F$ torch region, the magnetic vector potential $\mathrm{A}$ is determined from the following equations derived from Maxwell's equations [6]:

$$
\begin{aligned}
& \frac{1}{r} \frac{\partial}{\partial r}\left(r \frac{\partial A_{R}}{\partial r}\right)+\frac{\partial^{2} A_{R}}{\partial z^{2}}-\frac{A_{R}}{r^{2}}+\mu_{0} \omega \sigma A_{I}=0 \\
& \frac{1}{r} \frac{\partial}{\partial r}\left(r \frac{\partial A_{I}}{\partial r}\right)+\frac{\partial^{2} A_{I}}{\partial z^{2}}-\frac{A_{I}}{r^{2}}+\mu_{0} \omega \sigma A_{R}=0 .
\end{aligned}
$$

In the above equations, the subscripts $R$ and $I$ mean the real and the imaginary parts of the magnetic vector potential $\boldsymbol{A}$, respectively; i.e., $\boldsymbol{A}=A_{R}+i A_{I}$, where $i$ $=\sqrt{-1} . \quad \mu_{0}$ is the magnetic permeability of free space. According to Ampere's law and Faraday's law, the induced magnetic and electric fields, $\boldsymbol{B}^{\boldsymbol{R} \boldsymbol{F}}$ and $E^{R F}$, are, respectively, calculated as

$$
B_{z}^{R F}=\frac{1}{r} \frac{\partial}{\partial r}\left(r \frac{\partial A}{\partial r}\right), \quad B_{r}^{R F}=-\frac{\partial A}{\partial z},
$$


Table 2. Boundary conditions employed for the mathematical model of the DC- $R F$ hybrid plasma.

At the DC torch inlet $\left(z=-L_{D C}\right)$
$u=\frac{Q_{D C}}{\pi\left(R_{D C, 2}^{2}-R_{D C, 1}^{2}\right)}, \quad v=-u \tan (\pi / 4), \quad \omega=0, \quad T=300 \mathrm{~K}, \quad \frac{\partial A_{r}}{\partial z}=\frac{\partial A_{I}}{\partial z}=\frac{\partial \phi}{\partial z}=0$

At the anode inner surface of the $D C$ torch,

$u=v=w=0, \quad T=1000 \mathrm{~K}, \quad \frac{\partial A_{R}}{\partial z}=\frac{\partial A_{I}}{\partial z}=0, \quad \phi=0$

At the cathode surface of the DC torch

$u=v=w=0, T=3000 \mathrm{~K}$ (at the cathode spot), $\frac{\partial A_{R}}{\partial z}=\frac{\partial A_{I}}{\partial z}=0, j_{s}=j_{\max } \exp \left(-b \frac{\xi-\xi_{0}}{\xi_{0}-\xi_{s}}\right)$,

$j_{\max }$ and the radius of the cathode spot distributions $\xi-\xi_{0}$ are set to be $1.2 \times 10^{8} \mathrm{Am}^{-2}$ and 2 mm, respectively.

At the RF torch inlet $(z=0)$

$R_{1} \leq r \leq R_{2}: \quad u=v=w=0, \quad T=350 \mathrm{~K}, \quad \frac{\partial A_{R}}{\partial z}=\frac{\partial A_{I}}{\partial z}=0$

$R_{2} \leq r \leq R_{0}: \quad u=\frac{Q_{s}}{\pi\left(R_{0}^{2}-R_{2}^{2}\right)}, \quad v=0, \quad w=0, \quad T=350 \mathrm{~K}, \quad \frac{\partial A_{R}}{\partial z}=\frac{\partial A_{I}}{\partial z}=0$

At the centerline $(r=0)$

$\frac{\partial A_{u}}{\partial r}=\frac{\partial T}{\partial r}=\frac{\partial \phi}{\partial r}=v=w=A_{R}=A_{I}=0$

At the DC-RF hybrid torch exit $(z=L)$

$\frac{\partial(\rho u)}{\partial z}=\frac{\partial v}{\partial z}=\frac{\partial w}{\partial z}=\frac{\partial T}{\partial z}=\frac{\partial A_{R}}{\partial z}=\frac{\partial A_{I}}{\partial z}=\frac{\partial \phi}{\partial z}=0$

At the confinement tube wall $\left(r=R_{0}\right)$

$u=v=w=\frac{\partial \phi}{\partial z}=0, \quad T=350 \mathrm{~K}$

$\left.A_{r}\right|_{r=R_{0}}=\frac{\mu_{0}}{2 \pi}\left[\sum_{l} I_{c} \sqrt{\frac{R_{c, l}}{R_{0}}} G\left(k_{l}\right)+\sum_{i} \sum_{j} \omega \sigma_{i j} S_{i j} A_{I, i j} \sqrt{\frac{r_{j}}{R_{0}}} G\left(k_{i j}\right)\right]$

$\left.A_{I}\right|_{r=R_{0}}=-\frac{\mu_{0}}{2 \pi}\left[\sum_{i} \sum_{j} \omega \sigma_{i j} S_{i j} A_{R, i j} \sqrt{\frac{r_{j}}{R_{0}}} G\left(k_{i j}\right)\right]$

and

$$
E^{R F}=-i \omega A=-i 2 \pi f A,
$$

where $f$ is the $R F$ frequency applied to the induction coil of the $R F$ torch and $\omega=2 \pi f$. From these $R F$ - induced electric and magnetic fields, the Lorentz forces and the Joule heating can be derived from

$$
\begin{aligned}
& F_{z}^{R F}=-\frac{1}{2} \sigma R e\left[E^{R F} B_{r}^{R F *}\right], \\
& F_{r}^{R F}=+\frac{1}{2} \sigma R e\left[E^{R F} B_{z}^{R F *}\right], \\
& P_{j}^{R F}=\frac{1}{2} \sigma R e\left[E^{R F} E^{R F *}\right],
\end{aligned}
$$

where the superscript $*$ denotes the complex conjugate.

\section{B. Thermal Plasma Flow Equations}

The hybrid thermal plasma can be described by using the following conservation equations of mass, momentum and energy for laminar flow: i) Conservation of mass

$$
\frac{\partial}{\partial z}(\rho u)+\frac{1}{r} \frac{\partial}{\partial r}(r \rho v)=0
$$

ii) Conservation of momentum

$$
\begin{gathered}
\rho\left(u \frac{\partial u}{\partial z}+v \frac{\partial u}{\partial r}\right)=-\frac{\partial p}{\partial z}+\frac{1}{r} \frac{\partial}{\partial r}\left[\mu\left(\frac{\partial u}{\partial r}+\frac{\partial v}{\partial z}\right)\right] \\
+2 \frac{\partial}{\partial z}\left(\mu \frac{\partial u}{\partial z}\right)+F_{z}^{R F}+F_{z}^{D C}+\rho g
\end{gathered}
$$

axial component

$$
\begin{gathered}
\rho\left(v \frac{\partial v}{\partial z}+u \frac{\partial v}{\partial z}\right)=-\frac{\partial p}{\partial z}+\frac{1}{r} \frac{\partial}{\partial z}\left[\mu r\left(\frac{\partial u}{\partial r}+\frac{\partial v}{\partial z}\right)\right] \\
+\frac{2}{r} \frac{\partial}{\partial r}\left(\mu r \frac{\partial v}{\partial r}\right)-\frac{2 \mu v}{r^{2}}+\frac{\rho w^{2}}{r}+F_{r}^{R F}+F_{r}^{D C}
\end{gathered}
$$


radial component $(10)$

$$
\begin{aligned}
& \rho\left(v \frac{\partial w}{\partial r}+u \frac{\partial w}{\partial z}\right)=\frac{\partial}{\partial z}\left(\mu \frac{\partial w}{\partial z}\right)+\frac{1}{r} \frac{\partial}{\partial r}\left[\mu r \frac{\partial w}{\partial r}\right] \\
& -\frac{w}{r}\left(\rho v+\frac{\mu}{r}+\frac{\partial \mu}{\partial r}\right)
\end{aligned}
$$

azimuthal component

iii) Conservation of energy

$$
\begin{aligned}
& \rho\left(v \frac{\partial h}{\partial r}+u \frac{\partial h}{\partial z}\right)=\frac{\partial}{\partial z}\left(\frac{k}{C_{p}} \frac{\partial h}{\partial z}\right)+\frac{1}{r} \frac{\partial}{\partial r}\left(r \frac{k}{C_{p}} \frac{\partial h}{\partial r}\right) \\
& \quad+P^{R F}+P^{D C}-R^{0}
\end{aligned}
$$

where $u, v$ and $w$ are the axial, radial and azimuthal components of the plasma flow velocity and $\rho, p, \mu$ and $g$ represent the mass density, pressure, viscosity and gravity, respectively. In Eq. (11), $h$ is the plasma enthalpy, $k$ is the thermal conductivity and $C_{p}$ is the specific heat at constant pressure of the thermal plasma. $P^{R F}$ and $P^{D C}$ are the Joule heat generated by the $R F$ and the $D C$ input currents, respectively and $R^{0}$ is the volumetric radiation in the hybrid plasma.

\section{Boundary Conditions}

The full set of the boundary conditions imposed on the electromagnetic and flow equations is summarized in Table 2. Some boundary conditions listed in this table are adopted from the usual assumptions that have been widely used in thermal plasma science. For example, a constant electric potential $\phi$ is suggested at the anode surface of $D C$ plasma torches because the electrical conductivity of the anode nozzle conductor (normally $\mathrm{Cu}$ ) is assumed practically to be ideal. The explanations for the other boundary conditions used in this work can be found in our previous works, $[18,19]$.

\section{EQUIVALENT CIRCUIT AND IMPEDANCE CALCULATIONS}

The mechanism of $R F$ power transfer to a hybrid plasma can be interpreted as a transformer coupling between an induction coil and a hybrid plasma similar to an inductively coupled $R F$ plasma [6]. Figures 2(a) and (b) show the electric circuit diagrams of transformer coupling and its equivalent circuit, respectively, for the inductively coupled part of a $D C-R F$ hybrid plasma torch. In Figure 2, $V_{\text {torch }}$ is the voltage drop in the induction coil and $I, R$ and $L$ are the current, resistance and inductance, respectively, while the subscripts coil or $c, p$ and $e q$ designate the induction coil, plasma and equivalent value, respectively. From the basic electrical circuit theory, the $R F$ power $P_{0}$ dissipated in the plasma and the total magnetic power $P_{m a g}$ in the hybrid torch can be written in terms of the equivalent resistance $R_{e q}$ and the inductance $L_{e q}$ of the hybrid plasma as

$$
\begin{aligned}
& P_{0}=\frac{1}{2} I_{c}^{2}\left(R_{e q}-R_{c o i l}\right), \\
& P_{m a g}=\frac{1}{2} I_{c}^{2} \omega L_{e q} .
\end{aligned}
$$

The coupling efficiency, $\eta_{c}$ is defined as the ratio of the dissipated $R F$ power $P_{0}$ to the total magnetic power $P_{\text {mag }}[7]$ :

$$
\eta_{c} \equiv \frac{P_{0}}{P_{\text {mag }}}=\frac{R_{e q}-R_{c o i l}}{\omega L_{e q}} .
$$

If the coupling efficiency given in Eq. (16) is to be calculated, the equivalent resistance $R_{e q}$ and inductance $L_{e q}$ of a hybrid plasma should be known. In this paper, they are obtained from the voltage drop, $V_{\text {torch }}=\left(R_{e q}+\right.$ $\left.L_{e q}\right) I_{c}$, by using the following expression suggested by Kim et al. [12]:

$$
V_{\text {torch }}=\sum_{l}^{\text {coil }} 2 \pi R_{c, l}\left(\frac{I_{c}}{\sigma_{\text {coil }} S_{c o i l}}+i \omega A_{l}\right),
$$

where $\sigma_{\text {coil }}, S_{\text {coil }}$ and $A_{l}$ are the electrical conductivity of the coil conductor, the coil conduction area and the magnetic vector potential at the $l^{\text {th }}$ coil segment, respectively. The first term in the summation of the right-hand side of Eq. (17) is the voltage drop due to the coil resistance and the second one comes from the electromotive force induced at the $l^{t h}$ coil segment by the linkage of magnetic flux, which is generated by plasma currents and neighboring coil currents, as shown in Figure 2(c). Accordingly, the magnetic vector potential $A_{l}$ at the $l^{t h}$ coil is completely expressed by adding the contribution terms from neighboring coil currents, plasma currents and equivalent surface currents as

$$
\begin{aligned}
A_{l} & =\sum_{n}^{\text {coil }} \frac{I_{c}}{2 \pi} \Gamma_{n}-\sum_{m}^{\text {plasma }} j \frac{\mu_{0} \varpi}{2 \pi} \sigma_{m} A_{m} S_{m} \sqrt{\frac{r_{m}}{R_{c}}} G\left(k_{m}\right) \\
& +\sum_{s}^{\text {surface }} \frac{d z_{s}}{2 \pi} \sqrt{\frac{r_{m}}{R_{c}}}\left(\frac{\partial A_{s}}{\partial n} G\left(k_{s}\right)-A_{s} \frac{\partial G\left(k_{s}\right)}{\partial n}\right),(18)
\end{aligned}
$$

where $\Gamma_{n}$ is related to the self and the mutual inductances of coil segments and is suggested by Kim et al. [12] as follows:

$$
\begin{aligned}
& \Gamma_{n}=R_{c, l} \mu_{0} \sqrt{\frac{R_{c, n}}{R_{c, l}}} G\left(k_{l, n}\right), \quad \text { if } l \neq n ; \\
& \Gamma_{n}=10^{-9} N^{2} R_{c, l} P_{f} \text { if } l=n,
\end{aligned}
$$

where $P_{f}$ is the shape factor in Grover's self-inductance formula [20]. It should also be noted in Eq. (18) that $A_{l}$ includes all contributions from not only the current 
(a)

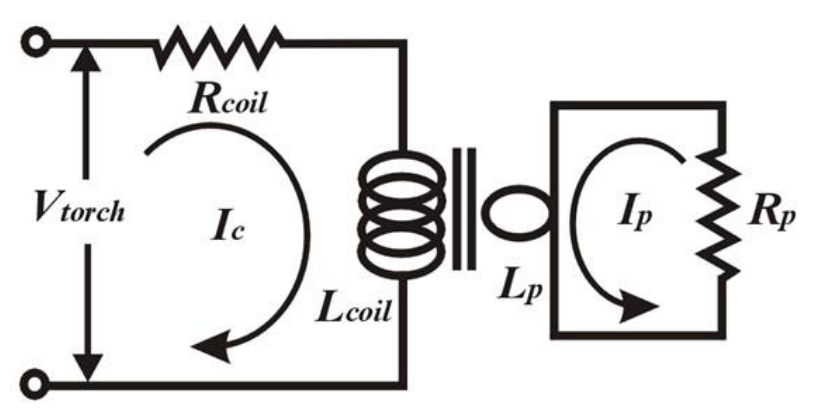

(b)

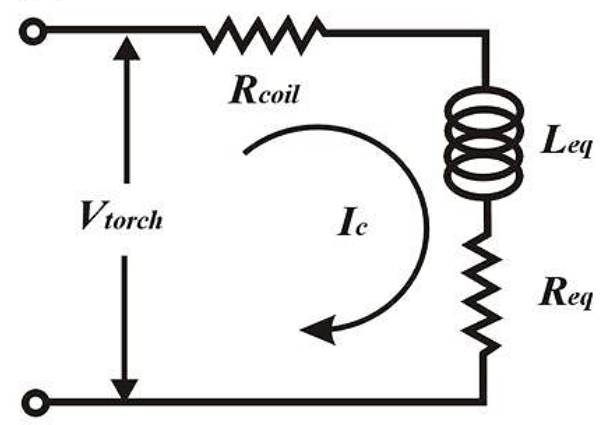

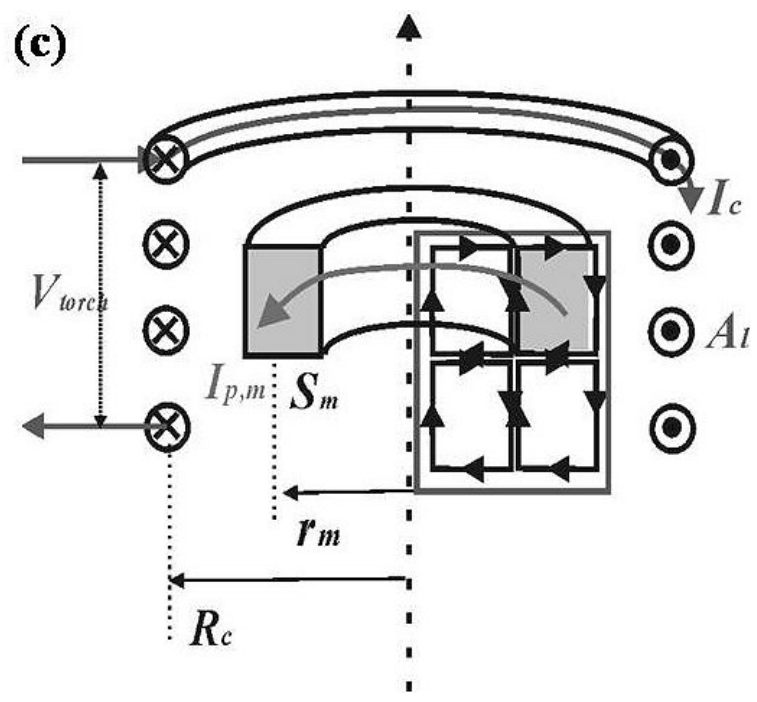

Fig. 2. (a) Transformer coupling diagram, (b) its equivalent circuit for the inductively coupled part of a $D C$ - $R F$ hybrid plasma torch and (c) a cross-sectional view of the induction coil segments and the plasma cells carrying a coil current Ic and an induced plasma current $I_{p, m}$.

sources of the plasma and neighboring coils but also the magnetic vector potentials at the plasma surfaces [17] due to the boundary conditions imposed at the wall, which is assumed to be generated by the equivalent surface current density at $R_{0}$ in Figure 1 . Including these contributions from the magnetic vector potentials at the plasma surfaces $\left(r=R_{0}\right)$ and substituting the expression for $A_{l}$ given in Eq. (18) into Eq. (17), the voltage drop $V_{\text {torch }}$ in the induction coil is rewritten as

$$
\begin{aligned}
& V_{\text {torch }}=\sum_{l}^{\text {coil }} 2 \pi R_{c, l}\left[\frac{I_{c}}{\sigma_{\text {coil }} S_{\text {coil }}}\right. \\
& +\frac{\mu_{0} \omega^{2}}{2 \pi} \sum_{m}^{\text {plasma }} \sigma_{m} \operatorname{Re}\left[A_{m}\right] r_{m} S_{m} \\
& \quad \times \sqrt{\frac{r_{m}}{R_{c, l}}} G\left(k_{m}\right)+\sum_{s}^{\text {surface }} \frac{\omega d z_{s}}{2 \pi} \sqrt{\frac{r_{s}}{R_{c, l}}} \\
& \left.\quad \times\left(\operatorname{Im}\left[A_{s}\right] \frac{\partial G\left(k_{s}\right)}{\partial n}-G\left(k_{s}\right) \frac{\partial \operatorname{Im}\left[A_{s}\right]}{\partial n}\right)\right]
\end{aligned}
$$

$$
\begin{aligned}
& +i \sum_{l}^{\text {coil }} 2 \pi R_{c, l}\left[\sum_{n}^{\text {coil }} \Gamma_{n}-\frac{\mu_{0} \omega^{2}}{2 \pi}\right. \\
& \times \sum_{m}^{\text {plasma }} \sigma_{m} \operatorname{Im}\left[A_{m}\right] r_{m} S_{m} \sqrt{\frac{r_{m}}{R_{c, l}}} G\left(k_{m}\right) \\
& +\sum_{s}^{\text {surface }} \frac{\omega d z_{s}}{2 \pi} \sqrt{\frac{r_{s}}{R_{c, l}}}\left(\operatorname{Re}\left[A_{s}\right] \frac{\partial G\left(k_{s}\right)}{\partial n}\right. \\
& \left.\left.-G\left(k_{s}\right) \frac{\partial \operatorname{Re}\left[A_{s}\right]}{\partial n}\right)\right]
\end{aligned}
$$

where $A_{m}$ and $A_{s}$ are the magnetic vector potentials from the $m^{t h}$ plasma cell and the $s^{t h}$ section of the confinement tube's wall, respectively. Re and Im mean the real and imaginary parts of magnetic vector potential.

By dividing the voltage drop $V_{\text {torch }}$ by the coil current $I_{c}$, the equivalent resistance $R_{e q}$ and inductance $L_{e q}$ for the hybrid plasma are, respectively, obtained as

$$
R_{e q}=\sum_{l}^{c o i l} 2 \pi R_{c, l}\left[\frac{1}{\sigma_{c o i l} S_{c o i l}}+\frac{\mu_{0} \omega^{2}}{2 \pi I_{c}}\right.
$$




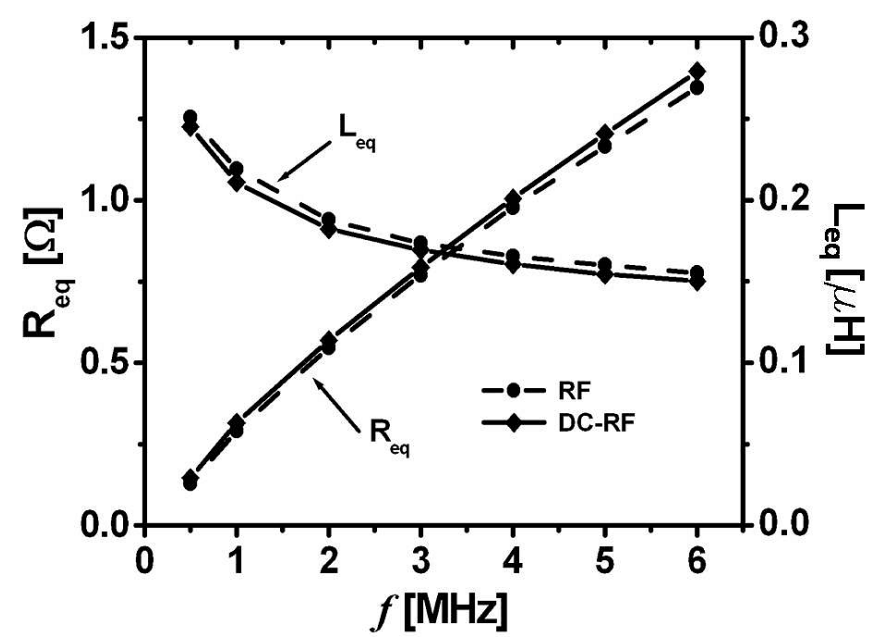

Fig. 3. Dependence of the equivalent resistance and the inductance on the $R F$ frequency for the $R F$ and the $D C-R F$ hybrid plasmas.

$$
\begin{gathered}
\times \sum_{m}^{\text {plasma }} \sigma_{m} \operatorname{Re}\left[A_{m}\right] r_{m} S_{m} \sqrt{\frac{r_{m}}{R_{c, l}}} G\left(k_{m}\right) \\
+\sum_{s}^{\text {surface }} \frac{\omega d z_{s}}{2 \pi I_{c}} \sqrt{\frac{r_{s}}{R_{c, l}}}\left(\operatorname{Im}\left[A_{s}\right] \frac{\partial G\left(k_{s}\right)}{\partial n}\right. \\
\left.\left.-G\left(k_{s}\right) \frac{\partial \operatorname{Im}\left[A_{s}\right]}{\partial n}\right)\right], \\
L_{e q}=\sum_{l}^{\text {coil }} 2 \pi R_{c, l}\left[\sum_{n}^{c o i l} \frac{\Gamma_{n}}{\omega}-\frac{\mu_{0} \omega}{2 \pi}\right. \\
\times \sum_{m}^{\text {plasma }} \sigma_{m} \operatorname{Im}\left[A_{m}\right] r_{m} S_{m} \sqrt{\frac{r_{m}}{R_{c, l}}} G\left(k_{m}\right) \\
+\sum_{s}^{\text {surface }} \frac{d z_{s}}{2 \pi} \sqrt{\frac{r_{s}}{R_{c, l}}}\left(\operatorname{Re}\left[A_{s}\right] \frac{\partial G\left(k_{s}\right)}{\partial n}\right. \\
\left.\left.-G\left(k_{s}\right) \frac{\partial R e\left[A_{s}\right]}{\partial n}\right)\right] .
\end{gathered}
$$

The first terms in the summations on the right-hand sides of Eqs. (21) and (22) represent the coil resistance and inductance, respectively. The second terms are contributions to the equivalent resistance and inductance from plasma currents and the last terms indicate the contributions of magnetic vector potentials at the wall's surface to those electrical parameters.

\section{RESULTS AND DISCUSSION}

\section{RF Frequency Effects}

In order to investigate the effect of the $R F$ frequency on the electrical coupling efficiency of the hybrid plasma,

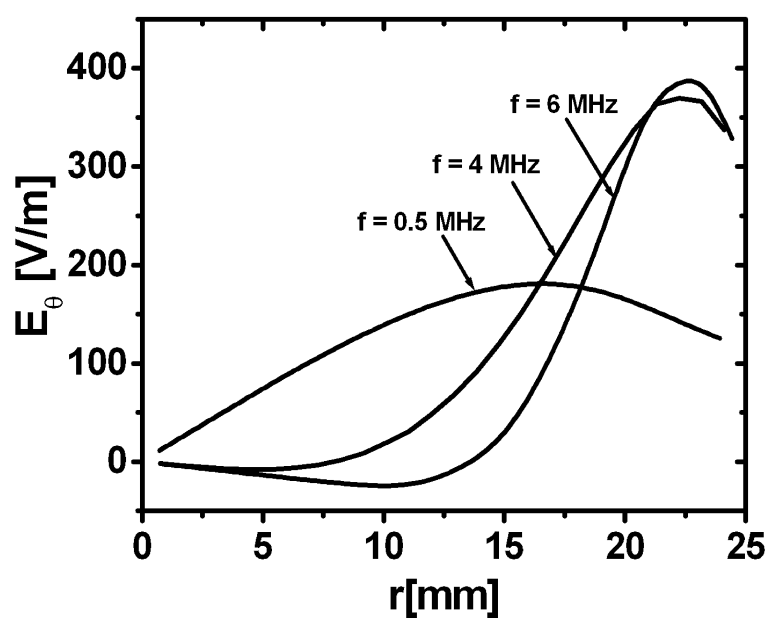

Fig. 4. Radial profiles of the electric field induced by different $R F$ frequencies $(f=0.5,4$ and $6 \mathrm{MHz})$ at the coil center $(z=60 \mathrm{~mm})$ in the $D C-R F$ hybrid plasma.

we carried out calculations for $R F$ frequencies from 0.5 to $6 \mathrm{MHz}$ and for a confinement tube's radius of $R_{0}=$ $25 \mathrm{~mm}$. The other design and operating conditions are fixed at the values listed in Table 1. Figure 3 shows the results calculated for the equivalent circuit parameters of the $D C-R F$ hybrid plasma compared with those of the $R F$ plasma. In this figure, the equivalent resistance $R_{e q}$ increases with the $R F$ frequency $f$ while the equivalent inductance $L_{e q}$ decreases. These behaviors of the equivalent parameters reflect the reduction of plasma skin depth $\delta$ by the increased frequency. As one can see in Figure 4 for the radial profiles of the electric fields induced by the $R F$ power input at the center $(z=60$ $\mathrm{mm}$ ) of the induction coil, higher electric fields tend to be distributed near the confinement tube wall at the higher frequency. Since plasma resistance is inversely proportional to the skin depth $\delta$, the equivalent resistance $R_{e q}$, which is the sum of the coil conductor and the plasma resistances, increases with $R F$ frequency $f$. Corresponding to the dependence of the electric field distributions on $R F$ frequency, the high temperature region of hybrid plasma is radially extended at high frequency to the confinement tube's wall, as illustrated in Figure 5. This expansion of the high temperature region brings about an increase in the flux linkage; consequently, the plasma inductance, $L_{p}$, increases with the $R F$ frequency. Normally, the equivalent inductance $L_{e q}$ is approximated by $L_{c o i l}-N^{2} L_{p}$ based on the basic electrical circuit theory for transformer coupling; accordingly, $L_{e q}$ should be decreased for higher $R F$ frequency, as in Figure 3, due to the increases in the flux linkage and the plasma inductance, $L_{p}$, which are accompanied by an expansion of the high- temperature region in the hybrid plasma. In Figure 5, the radial temperature distributions of the $D C-R F$ hybrid plasma in the coil zone turn out to be shifted more towards the confinement tube's wall than 


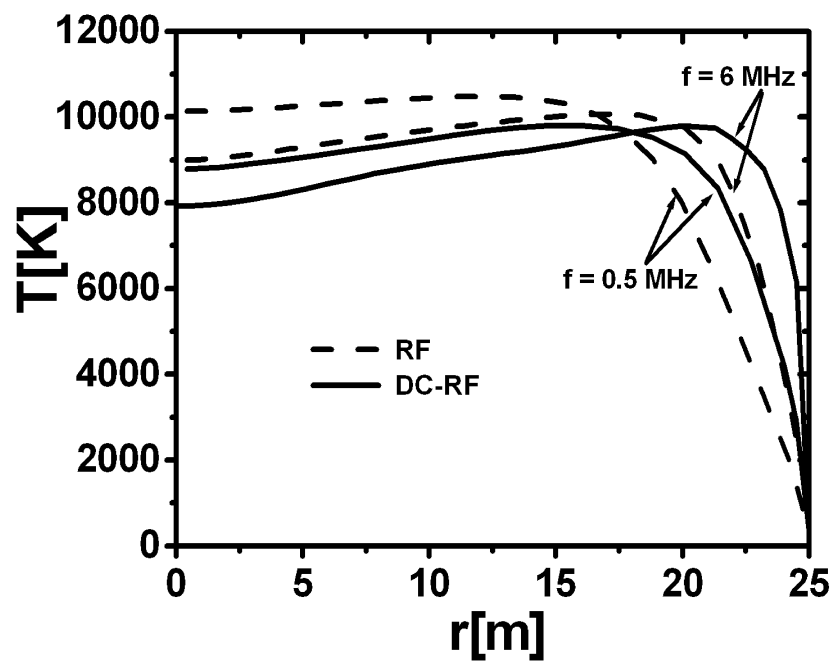

Fig. 5. Comparison of the radial profiles of the plasma temperature at the coil center $(z=60 \mathrm{~mm})$ between the $R F$ and the $D C$ - $R F$ hybrid plasmas with different $R F$ frequencies $(0.5$ and $6 \mathrm{MHz})$.

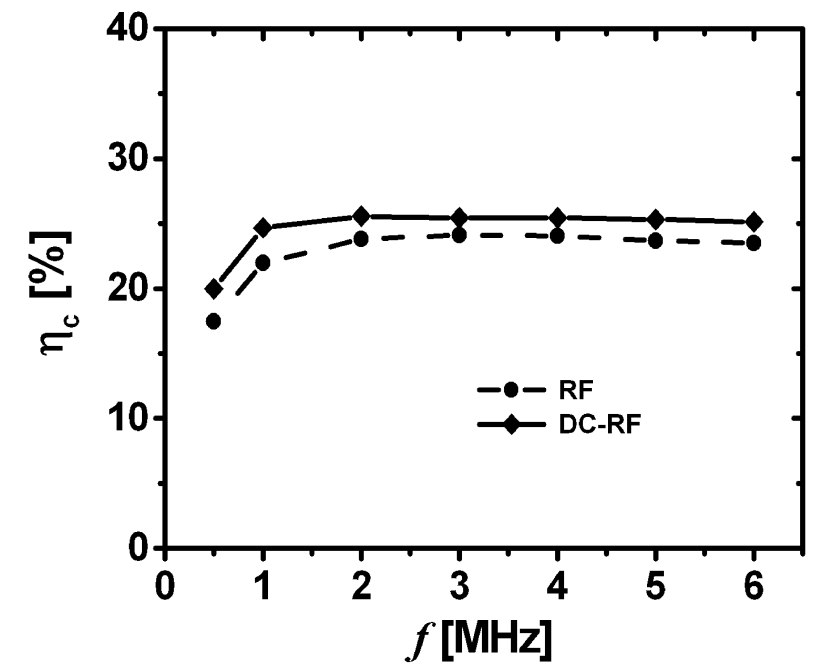

Fig. 6. Comparison of the dependence of the coupling efficiencies on the $R F$ frequency between the $R F$ and the $D C-R F$ hybrid plasmas.

those of the $R F$ plasma for $f=0.5$ and $4 \mathrm{MHz}$, which is caused by the presence of the $D C$ arc jet in the central region and the addition of its exit enthalpy to the hybrid plasma in the $R F$ torch region.

Figure 6 compares the electrical coupling efficiencies between the $R F$ and the $D C-R F$ hybrid plasmas for various $R F$ frequencies. In this figure, an almost constant small difference in the electrical coupling efficiency is observed in the frequency range from 2 to $6 \mathrm{MHz}$ due to the relatively linear dependency of the equivalent circuit parameters, $R_{e q}$ and $L_{e q}$, on the $R F$ frequency $f$, as shown in Figure 3. In addition, the electrical coupling efficiencies of the $D C-R F$ hybrid plasma are seen to be

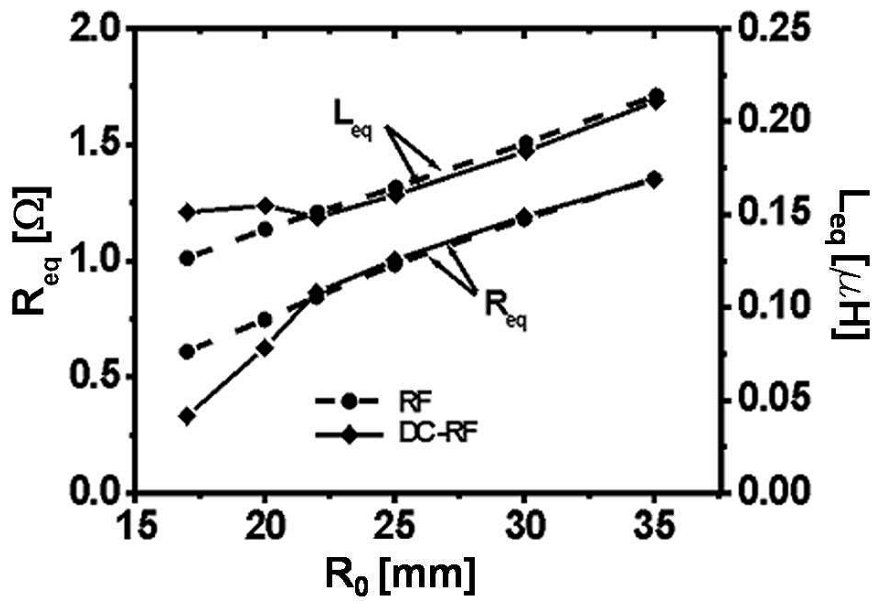

Fig. 7. Dependence of the equivalent resistance and inductance on the confinement tube's radius for the $R F$ and the $D C-R F$ hybrid plasmas.

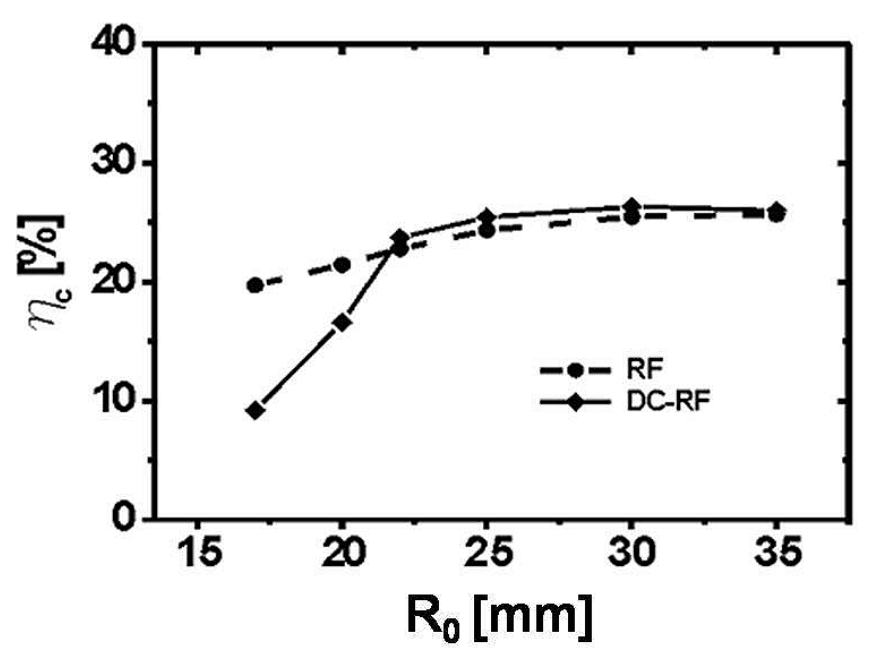

Fig. 8. Comparison of the dependence of the coupling efficiencies on the confinement tube's radius between the $R F$ and the $D C-R F$ hybrid plasmas.

slightly higher than those of the $R F$ plasma, which is the result of the $D C-R F$ hybrid plasma being more expanded toward the wall at the same $R F$ frequency, as observed in Figure 5.

\section{Confinement Tube Radius Effects}

To investigate the dependency of electric characteristics on the confinement tube's radius, we calculated the equivalent plasma resistance, $R_{e q}$, inductance, $L_{e q}$ and coupling efficiency, $\eta_{c}$, for a series of radius values and for the operation conditions given in Table 1. Figures 7 through 9 are plotted for the calculated electrical parameters, such as the equivalent resistance and inductance (Figure 7), the electrical coupling efficiency (Figure 8) 


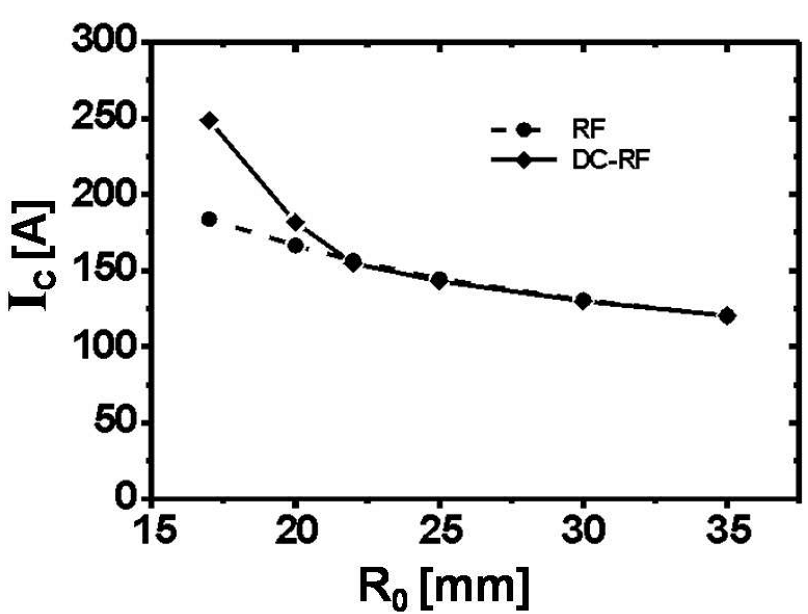

Fig. 9. Comparison of the dependence of the coil currents on the confinement tube's radius between the $R F$ and the $D C-R F$ hybrid plasmas.
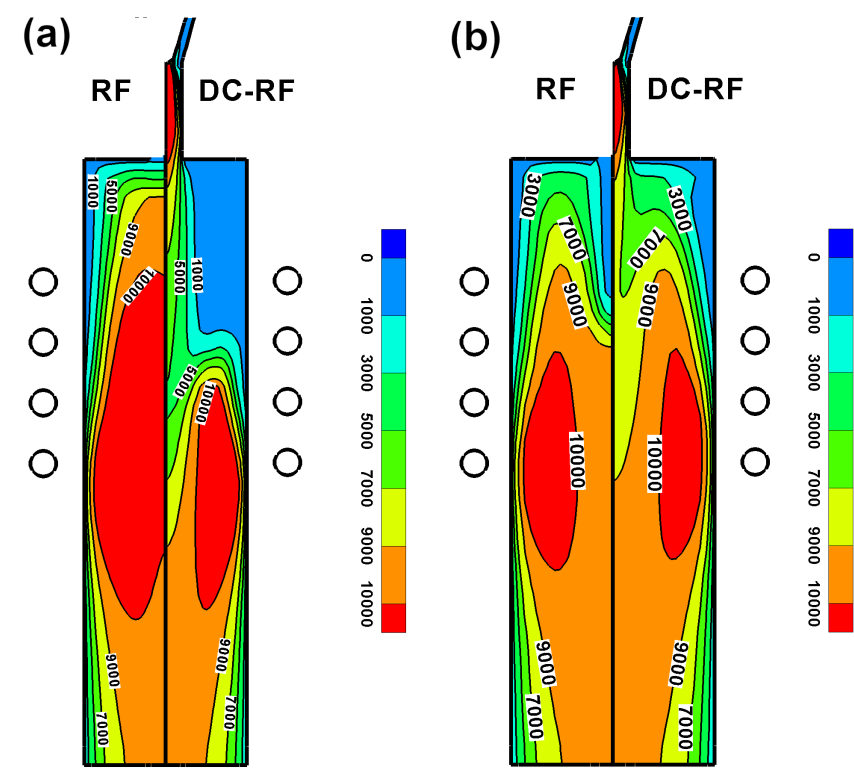

Fig. 10. Comparison of the temperature contours between the $R F$ and the $D C-R F$ hybrid plasmas with different confinement tube radii: (a) $R_{0}=20 \mathrm{~mm}$ and (b) $R_{0}=25 \mathrm{~mm}$.

and the coil currents (Figure 9), for various confinement tube's radius. Firstly, in Figure 7, the equivalent resistance and inductance increase linearly with the tube radius for both the $R F$ and the $D C-R F$ hybrid plasmas over the range from $R_{0}=22$ to $35 \mathrm{~mm}$. Such linear increases in $R_{e q}$ and $L_{e q}$ result directly from the multiplication effect of the coil radius $R_{c, l}$ accompanied by the change in the tube radius, as indicated in Eqs. (21) and (22). For the $D C-R F$ hybrid plasma, however, this linearity in $R_{e q}$ and $L_{e q}$ deviats over a small radius range from $R_{0}=17$ to $22 \mathrm{~mm}$. If the tube radius were to become smaller in this range, the $R_{e q}$ of the hybrid plasma would decrease more rapidly than that of the $R F$ plasma
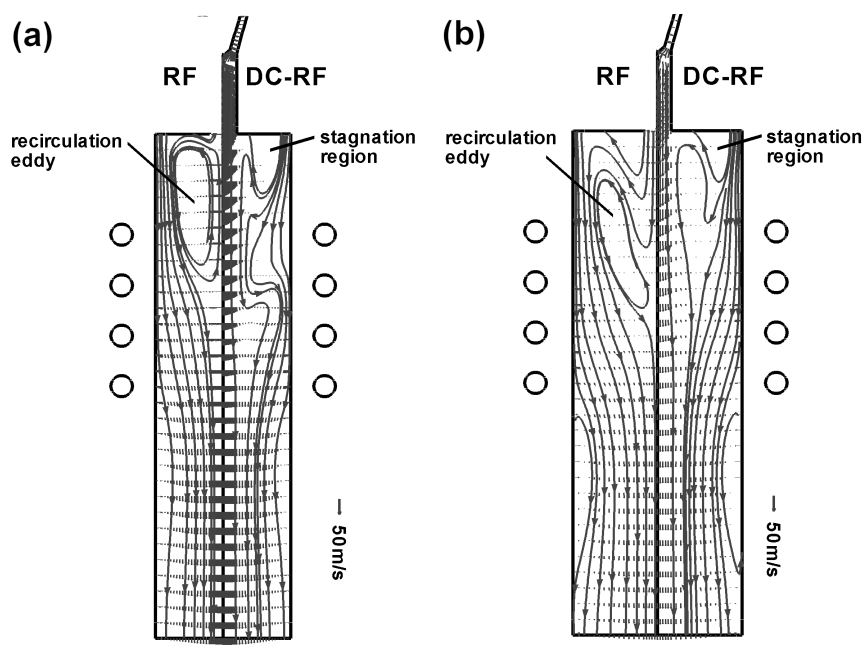

Fig. 11. Comparison of flow streamlines between the $R F$ and the $D C-R F$ hybrid plasmas with different confinement tube radii: (a) $R_{0}=20 \mathrm{~mm}$ and (b) $R_{0}=25 \mathrm{~mm}$.

while the $L_{e q}$ of the hybrid plasma would show values higher than that of the $R F$ plasma. Consequently, as the confinement tube's radius is reduced below $R_{0}=22 \mathrm{~mm}$, the coupling efficiency of the hybrid torch drastically deteriorate and the coil current rapidly increases compared with that of the $R F$ plasma, as shown in Figures 8 and 9. Such poor efficiency and increased coil currents for the $D C-R F$ hybrid plasma are attributed to a significant reduction in the high-temperature region upstream in the confinement tube for smaller radii, which is confirmed by comparing the right-hand temperature contours in Figures 10(a) and (b) for the hybrid plasma with $R_{0}=$ $20 \mathrm{~mm}$ and $25 \mathrm{~mm}$, respectively. As a result of the reduced high-temperature region, the magnetic flux linkage is also decreased for the smaller confinement tube, which leads to a drastic decrease in the electrical coupling efficiency of the $D C-R F$ hybrid plasma torch, as presented in Figure 8. In the case of the $R F$ plasma, however, the high-temperature region is simply redistributed upstream without much diminution for the smaller radius as seen in the left-hand temperature contours of Figures 10(a) and (b). As is well known in $R F$ plasma physics [4, $21,22]$, re-circulation eddies located in the upstream region of an $R F$ torch, which are illustrated in the left pictures of Figures 11(a) and (b), play an important role in sustaining the high-temperature plasma upstream. Basically, as long as these eddies exist, the high-temperature region in the upper part of the $R F$ torch is relatively unaffected by a reduction in the confinement tube's radius. As one can see in the right-hand streamlines of Figures $11(\mathrm{a})$ and (b) for the $D C-R F$ hybrid plasma with $R_{0}=20$ and $25 \mathrm{~mm}$, respectively, however, the re-circulation eddies are almost destroyed by a $D C$ arc jet and a small stagnation regime is formed under the $D C$ torch surrounded by a $D C$ arc jet in the central region and sheath gas flow in the peripheral region. This 
stagnation region is relatively more shifted towards the wall for $R_{0}=25 \mathrm{~mm}$ (see Figure 11(b) right), but it is contracted to the $D C$ arc jet as the confinement tube's radius becomes smaller (see Figure 11(a) right). From a comparison of these two figures, it is found that the streamlines of the stagnation region for $R_{0}=25 \mathrm{~mm}$ can play an role for convection heat transfer from the coil zone to the upper part of the confinement tube, but the ones for $R_{0}=20 \mathrm{~mm}$ show ineffective convection heat transfer by re-circulation of sheath gas flow heated in the coil region. As a result, a significant diminution of the high-temperature region upstream can be observed in Figure 10 (a) right. Consequently, a large reduction in the coupling efficiency can occur for a $D C-R F$ hybrid plasma torch with a small tube radius owing to the ineffective heating of the gas because of the contraction of the stagnation region under the $D C$ torch exit.

\section{CONCLUSION}

The electrical coupling efficiencies of inductivelycoupled $R F$ and $D C-R F$ hybrid plasma torches are numerically analyzed on the basis of thermal plasma flow and electrical circuit formulations to investigate their dependency on the design parameters, such as the $R F$ frequency and the confinement tube's radius. For this purpose, an integrated numerical model for the $D C-R F$ hybrid plasma, which couples the MHD equations to the appropriate boundary conditions for a $D C$ arc jet and an $R F$ flow over the entire region of the $D C-R F$ hybrid plasma torch, is presented and combined with an electrical circuit model for the impedance calculation method. We found from the calculated results for sufficiently large radii of the confinement tube, i.e., $R_{0}>22 \mathrm{~mm}$ in the present numerical illustrations, that the $R F$ frequency dependencies of the coupling efficiency and its associated equivalent electrical parameters show similar behaviors for both the $R F$ and the $D C-R F$ hybrid plasmas. Since the $R F$ frequency does not directly affect the overall generation of a high-temperature plasma, except for the skin depth region close to the tube's wall, we conclude that the electrical impedance of the hybrid plasma felt in the induction coil region shows results similar to those of the $R F$ plasma. However, if the confinement tube's radius decreases to less than some critical one, i.e., $R_{0}=$ $22 \mathrm{~mm}$ in the present numerical work, the coupling efficiency rapidly decreases for a $D C-R F$ hybrid plasma. Such poor efficiency of the hybrid torch with a relatively small radius results from a significant diminution of the high-temperature region upstream between the $D C$ torch exit and the first induction coil segment, which means that the reduced tube radius may lead to an ineffective coupling of the $D C$ arc jet and the $R F$ plasma. As a result of the reduced high-temperature region, the magnetic flux linkage is decreased for the smaller confinement tube, which leads to a drastic decrease in the electri- cal coupling. As the confinement tube's radius becomes smaller, the re-circulation eddies under the $D C$ torch are almost destroyed by a $D C$ arc jet and a stagnation region is formed and is contracted to the central region. From a comparison of the streamlines for two different confinement tube radii, the contraction of the stagnation region under the $D C$ torch exit and the resultant reduction of the high-temperature region are responsible for the poor coupling efficiency of the $D C-R F$ hybrid plasma torch. The contracted stagnation region prohibits convective heat transfer by re-circulation of the sheath gas flow from the coil zone to the upper part of the confinement tube, which ultimately results in a significant diminution of the high-temperature region upstream.

The present numerical analyses indicate that a special focus needs to be put on the influences of the $D C$ arc jet on the electrical and the flow characteristics of the $D C-R F$ hybrid plasma in determining the torch dimensions for effective conversion of $R F$ power into a plasma. Finally, we expect that the present numerical results to contribute to the design of $D C-R F$ hybrid torch systems. Furthermore, the numerical model developed in this work is expected to serve as a design tool for improving the performance of $D C-R F$ hybrid plasma torches.

\section{REFERENCES}

[1] T. Yoshida, T. Tani, H. Nishimura and K. Akashi, J. Appl. Phys. 54, 640 (1983).

[2] K. Yoshie, S. Kasuya, K. Eguchi and T. Yoshida, Appl. Phys. Lett. 61, 2782 (1992).

[3] X. H. Wang, K. Eguchi, C. Iwamoto and T. Yoshida, Sci. Technol. Adv. Mater. 3, 313 (2003).

[4] X. H. Wang, K. Eguchi, C. Iwamoto and T. Yoshida, Sci. Technol. Adv. Mater. 4, 159 (2003).

[5] R. Shimpo, Y. Uehara and T. Yoshida, Proceedings of 14th International Symposium on Plasma Chemistry IV (Praha, 1999), p. 2115.

[6] M. I. Boulos, Pure \& Appl. Chem. 57, 1321 (1985).

[7] K. Hisashi, S. Yoshinori and K. Shigeo, J. Plasma Fusion Res. 76, 731 (2000).

[8] M. P. Freeman and J. D. Chase, J. Appl. Phys. 39, 180 (1968).

[9] H. U. Eckert, J. Appl. Phys. 41, 1520 (1970).

[10] D. D. Hollister, Phys. Lett. 27A, 672 (1965).

[11] A. Chentouf, J. Fouladgar and G. Develey, IEEE Trans. Mag. 31, 2100 (1995).

[12] J. Kim, J. Mostaghimi and R. Iravani, IEEE Trans. Plasma Sci. 25, 1023 (1997).

[13] A. Merkhouf and M. I. Boulos, Plasma Sources Sci. Technol. 7, 599 (1998).

[14] A. Merkhouf and M. I. Boulos, J. Phys. D: Appl. Phys. 33, 1581 (2000).

[15] S. V. Patankar, Computational Fluid Flow and Heat Transfer (McGraw-Hill, New York, 1980).

[16] J. Mostaghimi and M. I. Boulos, Plasma Chem. Plasma Process. 9, 25 (1989).

[17] J. A. Tegopoulos and E. E. Kriezis, Eddy Currents in Linear Conducting Media (Elsevier, Amsterdam, 1985). 
[18] J. H. Seo, J. M. Park and S. H. Hong, Plasma Sources Sci. Technol. 17, 025011 (2008).

[19] J. H. Seo, Ph.D. dissertation, Seoul National University, 2004.

[20] F. W. Grover, Inductance Calculations (Van Nostrand, New York, 1946), p. 95.
[21] J. H. Park and S. H. Hong, J. Korean Phys. Soc. 31, 753 (1997).

[22] M. I. Boulos, R. Gagne and R. M. Barns, Can. J. Chem. Eng. 58, 367 (1980). 